\title{
Reclassification of Whole Exome Sequencing-derived Genetic Variants in Pendred Syndrome with ACMG/AMP Standards
}

\author{
Kok-Siong Poon ${ }^{1}$ Karen Mei-Ling $\operatorname{Tan}^{1}$ \\ ${ }^{1}$ Department of Laboratory Medicine, National University Hospital \\ Singapore, Singapore, Singapore
}

Global Med Genet 2021;8:129-131.
Address for correspondence Kok-Siong Poon, MSc, Department of Laboratory Medicine, National University Hospital, 5 Lower Kent Ridge Rd, 119074 Singapore (e-mail: kok_siong_poon@nuhs.edu.sg).
Whole exome sequencing (WES) opens up an unbiased testing approach for genetic diagnosis of rare disorders compared with single-gene and multigene panel testing. Being a diagnostic tool for a wide range of genetic diseases, WES identifies causal variants in close to one-third of the referred cases, and variants of unknown significance (VUS) potentially responsible for the clinical presentations in another one quarter of index patients. ${ }^{1}$ Systematic guidelines from Human Genome Variation Society (HGVS), and American College of Medical Genetics and Genomics (ACMG) and Association for Molecular Pathology (AMP) are currently available for standardizing the nomenclature and reporting the interpretation of genetic variants, respectively. ${ }^{2,3}$ Despite this, variant curation still remains a challenge in the face of advances in sequencing technologies, which may reveal large numbers of novel variants possibly associated with the clinical phenotype in the diagnostic setting. Depending on the disease context, reanalysis of WES-derived genetic variants can sometimes improve diagnostic yields ${ }^{4}$ or result in downgrading of the pathogenicity status of some previously reported variants. ${ }^{5}$

Pendred syndrome (OMIM 274600) is an autosomal recessive disorder characterized by sensorineural hearing loss, goiter, and inner ear malformations. It is most commonly caused by biallelic loss-of-function variants in the SLC26A4 gene. ${ }^{6}$ Digenic inheritance of monoallelic loss-of-function variants in the SLC26A4 gene and another gene, for example, either KCNJ10, FOXI1 or GJB2 have also been described. ${ }^{7-9}$ However, subsequent studies refuted the causal associations between KCNJ10 or FOXI1 mutations and SLC26A4 mutations in this syndrome. ${ }^{10,11}$

Here, we demonstrated the reclassification of causative variants previously reported in a study ${ }^{11}$ in which WES was performed on a family with two daughters clinically diagnosed with Pendred syndrome and their unaffected parents. ${ }^{12}$ On the basis of possible autosomal recessive, digenic or oligogenic modes of inheritance, pathogenic variants were sought in the two sisters. Thyroid dysfunction in the two siblings was attributed to compound heterozygous variants (p.Lys350* and p.Arg1110Gln) in the DUOX2 gene. Interestingly, Chow et al proposed that the combination of heterozygous variants in three genes (SLC26A4 p.Ser448Leu, GJB2 p.Thr123Asn, and SCARB2 p.Thr305Met), which were found in both affected siblings, led to bilateral hearing loss.

Digenic inheritance usually involves pathogenic variants in genes encoding interacting proteins, and results in a "double hit" affecting the function or structure of the protein complex. ${ }^{13}$ Notably, the three genes reported by Chow et al, namely, SLC26A4, GJB2 and SCARB2, have no known protein interactions between their gene products. In Chow et al, the evidence of pathogenicity for the candidate variants in these genes were derived solely from in silico predictions, without evidence from functional analyses or gene-disease associations other than from the single family. This prompted us to further curate these variants according to the ACMG/AMP standards ${ }^{3}$ using VarSome. ${ }^{14}$ We also performed data mining in ClinVar, a database commonly referenced for germline variants. ${ }^{15}$ The pathogenicity of the variants identified in the two siblings with Pendred syndrome in Chow et al are shown in - Table 1. After re-evaluation, the SLC26A4 NM_000441.2: c.1343C $>\mathrm{T}$ variant was classified as "pathogenic" and "likely pathogenic" by ACMG/AMP and ClinVar, respectively. Strikingly, the GJB2 NM_004004.6:c.368C >A and SCARB2 NM_005506.4:c.914C > T variants were classified as either benign or VUS. The GJB2 p.Thr123Asn variant has been detected at higher frequencies in controls compared with patients ${ }^{16}$ and identified in trans with pathogenic variants in the GJB2 gene in normal carriers. ${ }^{17}$ Taking these reevaluations into consideration, the GJB2 and SCARB2 variants are now "downgraded" in their pathogenicity status and unlikely to be associated with deafness in concert with the SLC26A4 variant. published online March 10, 2021
DOI https://doi.org/ $10.1055 / \mathrm{s}-0041-1725072$. ISSN 2699-9404. (c) 2021. The Author(s).

This is an open access article published by Thieme under the terms of the Creative Commons Attribution License, permitting unrestricted use, distribution, and reproduction so long as the original work is properly cited. (https://creativecommons.org/licenses/by/4.0/)

Georg Thieme Verlag KG, Rüdigerstraße 14, 70469 Stuttgart, Germany 
Table 1 Classification of variants according to the ACMG/AMP standards and ClinVar

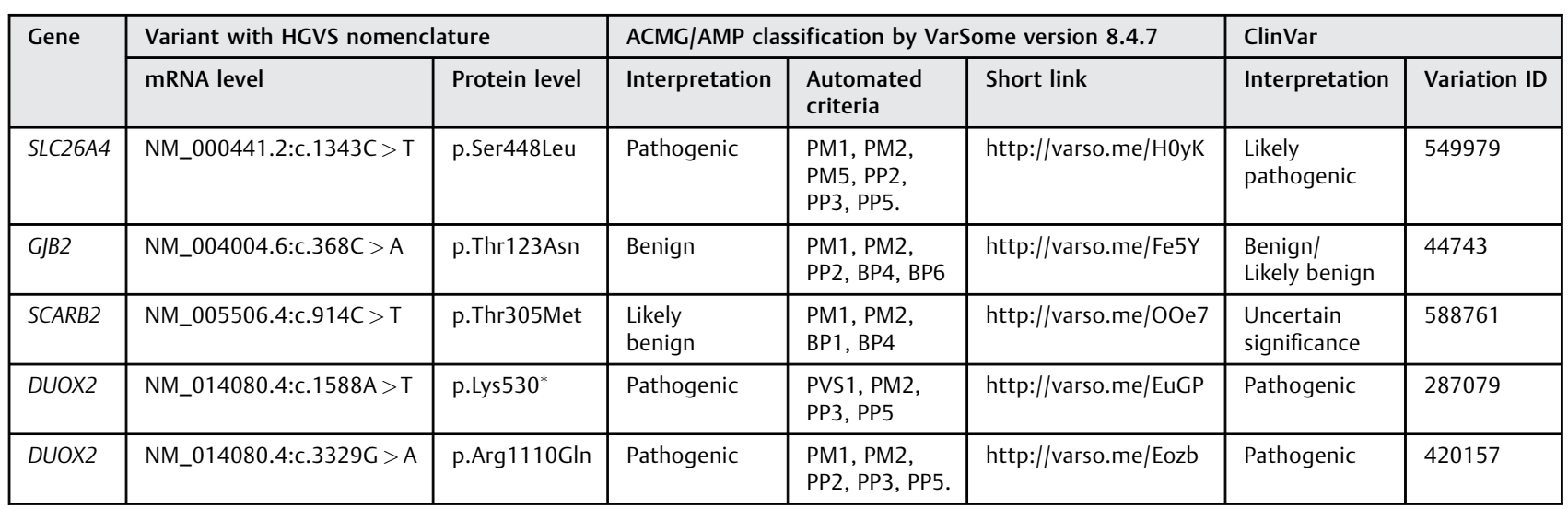

On the other hand, the two DUOX2 variants, namely, NM_014080.4:c.1588A $>$ T and c.3329G $>$ A were classified as "pathogenic" in the context of goiter by ACMG/AMP standards and in ClinVar. These classifications are consistent with Chow et al's interpretations. The two pathogenic variants in the DUOX2 gene identified (p.Lys350* and p. Arg1110Gln) have been found in patients with congenital hypothyroidism. $^{18-20}$

WES investigates all coding exons in a relatively timeefficient and cost-effective manner compared with the traditional single-gene or multigene panel testing. It is not unusual that many rare variants can be identified in multiple genes by WES. It remains a big challenge to firmly assign causal variants and genes when there is only one individual or a single family, especially in complex diseases with possibly digenic or polygenic inheritance. There are many essential points to consider when evaluating the pathogenicity of variants ${ }^{3}$ and also when conducting reevaluation and reanalysis of genomic test results. ${ }^{21}$ Having timely and accurately updated public databases of variants is essential to the research and clinical diagnostic communities for variant curation and clinical diagnosis.

Funding

None.

\section{Conflict of Interest}

None declared.

\section{References}

1 Trujillano D, Bertoli-Avella AM, Kumar Kandaswamy K, et al. Clinical exome sequencing: results from 2819 samples reflecting 1000 families. Eur J Hum Genet 2017;25(02):176-182

2 den Dunnen JT, Dalgleish R, Maglott DR, et al. HGVS recommendations for the description of sequence variants: 2016 update. Hum Mutat 2016;37(06):564-569

3 Richards S, Aziz N, Bale S, et al; ACMG Laboratory Quality Assurance Committee. Standards and guidelines for the interpretation of sequence variants: a joint consensus recommendation of the American College of Medical Genetics and Genomics and the Association for Molecular Pathology. Genet Med 2015;17(05):405-424
4 Salfati EL, Spencer EG, Topol SE, et al. Re-analysis of whole-exome sequencing data uncovers novel diagnostic variants and improves molecular diagnostic yields for sudden death and idiopathic diseases. Genome Med 2019;11(01):83

5 Campuzano O, Sarquella-Brugada G, Fernandez-Falgueras A, et al. Reanalysis and reclassification of rare genetic variants associated with inherited arrhythmogenic syndromes. EBioMedicine 2020; 54:102732

6 Bizhanova A, Kopp P. Genetics and phenomics of Pendred syndrome. Mol Cell Endocrinol 2010;322(1-2):83-90

7 Yang T, Vidarsson H, Rodrigo-Blomqvist S, Rosengren SS, Enerback S, Smith RJ. Transcriptional control of SLC26A4 is involved in Pendred syndrome and nonsyndromic enlargement of vestibular aqueduct (DFNB4). Am J Hum Genet 2007;80(06): 1055-1063

8 Yang T, Gurrola JG II, Wu H, et al. Mutations of KCNJ10 together with mutations of SLC26A4 cause digenic nonsyndromic hearing loss associated with enlarged vestibular aqueduct syndrome. Am J Hum Genet 2009;84(05):651-657

9 Ben Said M, Dhouib H, BenZina Z, et al. Segregation of a new mutation in SLC26A4 and p.E47X mutation in GJB2 within a consanguineous Tunisian family affected with Pendred syndrome. Int J Pediatr Otorhinolaryngol 2012;76(06):832-836

10 Landa P, Differ AM, Rajput K, Jenkins L, Bitner-Glindzicz M. Lack of significant association between mutations of KCNJ10 or FOXI1 and SLC26A4 mutations in Pendred syndrome/enlarged vestibular aqueducts. BMC Med Genet 2013;14:85

11 Zhao J, Yuan Y, Huang S, et al. KCNJ10 may not be a contributor to nonsyndromic enlargement of vestibular aqueduct (NSEVA) in Chinese subjects. PLoS One 2014;9(11):e108134

12 Chow YP, Abdul Murad NA, Mohd Rani Z, et al. Exome sequencing identifies SLC26A4, GJB2, SCARB2 and DUOX2 mutations in 2 siblings with Pendred syndrome in a Malaysian family. Orphanet J Rare Dis 2017;12(01):40

13 Schäffer AA. Digenic inheritance in medical genetics. J Med Genet 2013;50(10):641-652

14 Kopanos C, Tsiolkas V, Kouris A, et al. VarSome: the human genomic variant search engine. Bioinformatics 2019;35(11): 1978-1980

15 Landrum MJ, Lee JM, Benson M, et al. ClinVar: improving access to variant interpretations and supporting evidence. Nucleic Acids Res 2018;46(D1):D1062-D1067

16 Dai P, Yu F, Han B, et al. GJB2 mutation spectrum in 2,063 Chinese patients with nonsyndromic hearing impairment. J Transl Med 2009;7:26

17 He L, Pang X, Chen P, Wang X, Yang T, Wu H. Carrier re-sequencing reveals rare but benign variants in recessive deafness genes. Sci Rep 2017;7(01):11355 
18 Fu C, Zhang S, Su J, et al. Mutation screening of DUOX2 in Chinese patients with congenital hypothyroidism. J Endocrinol Invest 2015;38(11):1219-1224

19 Maruo Y, Takahashi H, Soeda I, et al. Transient congenital hypothyroidism caused by biallelic mutations of the dual oxidase 2 gene in Japanese patients detected by a neonatal screening program. J Clin Endocrinol Metab 2008;93(11): 4261-4267
20 Fu C, Luo S, Zhang S, et al. Next-generation sequencing analysis of DUOX2 in 192 Chinese subclinical congenital hypothyroidism (SCH) and $\mathrm{CH}$ patients. Clin Chim Acta 2016;458:30-34

21 Deignan JL, Chung WK, Kearney HM, Monaghan KG, Rehder CW, Chao ECACMG Laboratory Quality Assurance Committee. Points to consider in the reevaluation and reanalysis of genomic test results: a statement of the American College of Medical Genetics and Genomics (ACMG). Genet Med 2019;21(06):1267-1270 